\title{
Hypervelocity impact phenomena of LPSO-magnesium alloys
}

\author{
Masahiro Nishida ${ }^{1, *}$, Fumiya Kodama $^{1}$, Koichi Hayashi ${ }^{2}$, Yasuhiro Akahoshi ${ }^{3}$, \\ Kazuyuki Hokamoto ${ }^{4}$, and Yoshihito Kawamura ${ }^{4}$ \\ ${ }^{1}$ Nagoya Institute of Technology, Gokiso-cho, Showa-ku, Aichi, 466-8555 Japan \\ ${ }^{2}$ National Institute of Technology, Toba College, 1-1, Ikegami-cho, Toba City, Mie, 517-8501 Japan \\ ${ }^{3}$ Kyushu Institute of Technology, 1-1 Sensui-cho, Tobata-ku, Kitakyushu-shi, Fukuoka, 804-8550 Japan \\ ${ }^{4}$ Kumamoto University, 2-39-1 Kurokami, Chuo-ku, Kumamoto, 860-8555, Japan
}

\begin{abstract}
Hypervelocity impact phenomena of long period stacking ordered type magnesium alloys (LPSO-Mg) plates were examined at the impact velocities of $4 \mathrm{~km} / \mathrm{s}$ and $6 \mathrm{~km} / \mathrm{s}$. Ejecta veil and external bubble of debris of LPSO-Mg were darker than those of aluminum alloy A6061-T6. The size of external bubble of debris of LPSO-Mg was slightly smaller than that of A6061-T6. The velocity reduction of LPSO$\mathrm{Mg}$ was slightly larger than that of A6061-T6. However, the scatter area of projectile and targets were not determined by electron probe micro-analyzer.
\end{abstract}

\section{Introduction}

The International Space Station (ISS) employs shields such as the Whipple bumper to protect from micrometeoroids and orbital debris (MMOD). The main bumper of ISS consists of aluminum alloy thin plates. To improve shielding efficiency, several studies using foam core sandwich panels [1-3], cellular structures [4], hybrid metal foams [5], ceramic plates [6], amorphous alloy reinforced aluminum alloy plates [7], and types of metallic glass [8-9] have been carried out.

Magnesium alloys show promise for space applications because of several advantages such as low density and high vibration absorption. New types of magnesium alloys using rare metals overcame longtime disadvantages of conventional magnesium alloys of low strength. Among them, magnesium alloys with long period stacking order (LPSO) phase and alpha phase with high strength have been developed [10, 11]. Many industrial applications such as automobiles, aircraft, and spacecraft have been being considered. In the case of space application, the impact resistance against space debris is very important because the typical impact velocity of space debris is around $10 \mathrm{~km} / \mathrm{s}$.

In this study, penetration phenomena of LPSO-type magnesium alloy plates were studied in hypervelocities. Pressure walls after impact experiments were observed. Results of LPSO-type magnesium alloy plates were compared with those of aluminum alloy plates.

\section{Experimental methods}

Plates of LPSO-type magnesium alloy, Mg95.65Zn1Y2La0.1A10.25 (at. \%) (Fuji Light Metal Co.), were used as targets (hereinafter referred to as LPSO-Mg). Aluminum alloy A6061-T6 plates were also used as target materials to compare with the results of LPSO-Mg. The target size was $150 \mathrm{~mm}$ in length and 50 $\mathrm{mm}$ in width. The thickness of LPSO-Mg was $1.5 \mathrm{~mm}$, and the aluminum alloy was $1.0 \mathrm{~mm}$. The thickness was chosen so that the areal density was almost the same. The density of the magnesium alloy was $1.86 \mathrm{~g} / \mathrm{cm}^{3}$, and that of the aluminum alloy was $2.70 \mathrm{~g} / \mathrm{cm}^{3}$. In this study, LPSO-Mg plates and aluminum alloy plates with the same areal density were examined in terms of the basic properties of penetration mechanism to be considered in space application as substitute bumper materials.

Projectiles were made of aluminum alloy 2017-T4 with a diameter of $3.2 \mathrm{~mm}$. The impact velocity was 5.0 $\mathrm{km} / \mathrm{s}$. Two-stage light gas guns at the Japan Aerospace Exploration Agency (JAXA) were used for the impact test [3]. The debris cloud immediately after impact was observed using a high-speed video camera (Shimadzu, HPV-X).

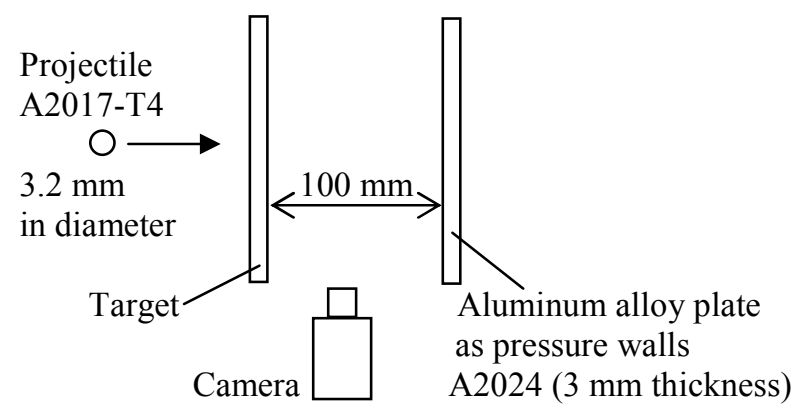

Fig 1. Setup for penetration experiments.

\section{Results}

Figure 1 is shadowgraph images of the high speed video camera. We could observe clear ejecta veil, external

Corresponding author: nishida.masahiro@nitech.ac.jp 
bubble of debris, and internal structure of debris cloud in the case of LPSO-Mg as well as A6061-T6 of previous works $[12,13]$ and A6061-T6 of our results. In the case of LPSO-Mg, the front element of the internal structure was not clear. The size of the external bubble of LPSO$\mathrm{Mg}$ seemed to be slightly smaller than that of aluminum alloy. The debris cloud of LPSO-Mg was significantly darker than that of A6061-T6. In particular, ejecta veil of LPSO-Mg was darker than that of A6061-T6.

Next, the velocity of the front element was compared with the impact velocity of the projectile using a high speed video camera. The velocities were calculated from seven flames (the impact velocity of $6 \mathrm{~km} / \mathrm{s}$ ) and two flames (the impact velocity of $4 \mathrm{~km} / \mathrm{s}$ ) before and after impact using image analysis software. In the case of A6061-T6, the velocity of the front element was 0.88 $\mathrm{km} / \mathrm{s}$ lower than the impact velocity of the projectile in Figure $2 \mathrm{a}$. In the case of LPSO-Mg, the velocity of the front element was $1.30 \mathrm{~km} / \mathrm{s}$ lower than the impact velocity of the projectile in Figure $2 \mathrm{~b}$. In Figure 2c for A6061-T6, the velocity of the front element was 0.78 $\mathrm{km} / \mathrm{s}$ lower than the impact velocity of the projectile. In Figure $2 \mathrm{~d}$ for LPSO-Mg, the velocity of the front element was $1.0 \mathrm{~km} / \mathrm{s}$ lower than the impact velocity of the projectile. The velocity reduction decreased with increasing impact velocity. This means that the velocity of the front element approached the impact velocity with increasing impact velocity. In both materials, the velocity of the front element and the size of the external bubble of debris increased with impact velocity. This tendency was the same in previous works $[12,13]$. The reduction velocity of the front element for LPSO-Mg was greater than that for A6061-T6. It is a good property for bumper materials.

Debris cloud impact patterns on aluminum alloy plates placed $100 \mathrm{~mm}$ behind each target plate were observed after impact experiments. In both cases, we can clearly see a white circle in the center of the aluminum alloy plates, which were made by the impact of the front element of the debris cloud. In the case of aluminum alloy, we can see clear radial lines that are a typical of a debris cloud impact pattern [14], whereas in the case of LPSO-Mg the radial lines become less clear. Instead, we can see a larger white circle like a fine mist.

Weight percentage of aluminum and magnesium at each measure point were analysed by an electron probe micro-analyzer (EPMA, JEOL JXA-8230). Six small plates $(15 \mathrm{~mm}$ by $15 \mathrm{~mm})$ were place on the aluminum alloy plates to be able to be measured by the analyzer. The measured diameter of each plate was $20 \mu \mathrm{m}$. Tables 1 and 2 show results of A6061-T6 and LPSO-Mg, respectively. Table 1 shows that weight percentage of magnesium was $2-5 \%$ even though the target was aluminum alloy. The main reason for this is that the target material, projectile material, and pressure wall materials include less than $2 \%$ magnesium. In the case of LPSO-Mg targets, Table 2 shows that weight percentage of magnesium was $5-12 \%$. At the measured point 4 , which is $40 \mathrm{~mm}$ away from the centre of the debris cloud impact patterns, weight percentage of magnesium was $12 \%$. It was expected from the size of the debris cloud in
Figure 2 that results of point 5 would show much magnesium, but the result was contrary to expectation.

The scatter area of projectile and targets were not determined by electron probe micro-analyzer.

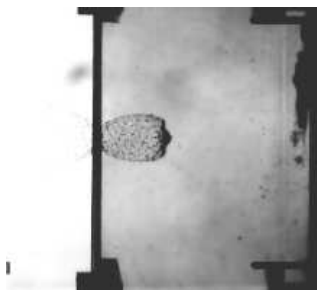

(a-1) $10 \mu$ s after impact

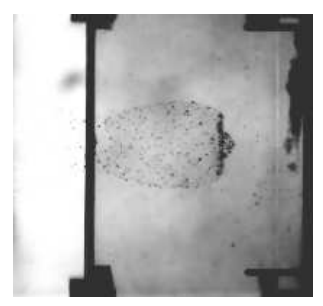

(a-2) $20 \mu$ s after impact (a) $\operatorname{A6061-T6~}(4.17 \mathrm{~km} / \mathrm{s})$

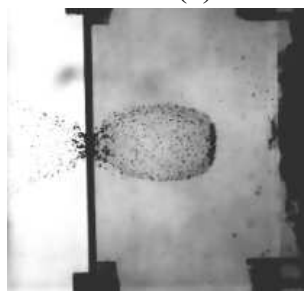

(b-1) $10 \mu$ s after impact

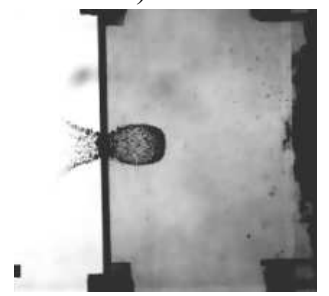

(b-2) $20 \mu$ s after impact (b) LPSO-Mg $(4.10 \mathrm{~km} / \mathrm{s})$

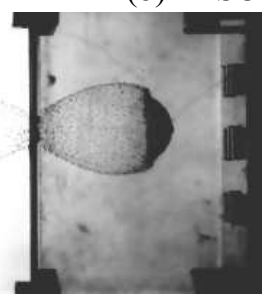

(c-1) $7.5 \mu$ s after impact

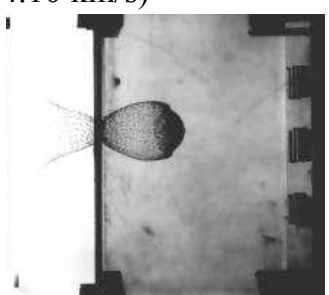

(c-2) $12.5 \mu \mathrm{s}$ after impact

$6.08 \mathrm{~km} / \mathrm{s})$

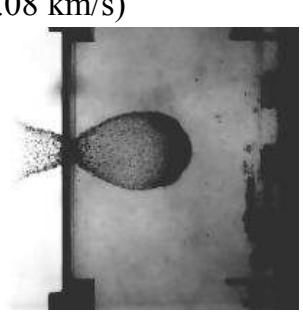

(d-2) $12.5 \mu \mathrm{s}$ after (d-1) $7.5 \mu \mathrm{s}$ after impact impact

(d) LPSO-Mg $(6.11 \mathrm{~km} / \mathrm{s})$

Fig. 2. Shadowgraph images of high speed video camera.

Table 1. Weight percentage of aluminum and magnesium for A6061-T6 target.

\begin{tabular}{|c|c|c|c|c|c|c|}
\hline Element & Point 1 & Point 2 & Point 3 & Point 4 & Point 5 & Point 6 \\
\hline $\mathrm{Al}$ & 74.7 & 78.4 & 83.5 & 86.4 & 80.1 & 83.4 \\
\hline $\mathrm{Mg}$ & 5.5 & 2.7 & 2.6 & 2.7 & 2.5 & 2.4 \\
\hline
\end{tabular}

Table 2. Weight percentage of aluminum and magnesium at each measure points for LPSO-Mg target.

\begin{tabular}{|c|c|c|c|c|c|}
\hline Element & Point 1 & Point 2 & Point 3 & Point 4 & Point 5 \\
\hline $\mathrm{Al}$ & 73.0 & 77.1 & 73.0 & 67.0 & 73.1 \\
\hline $\mathrm{Mg}$ & 7.1 & 5.4 & 6.3 & 12.6 & 7.5 \\
\hline
\end{tabular}




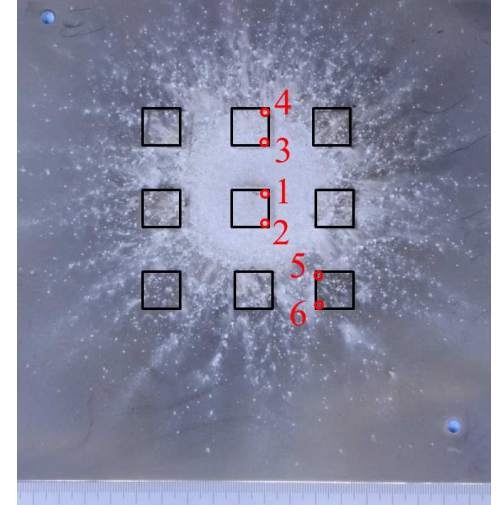

(a) A6061-T6 (6.08 km/s)

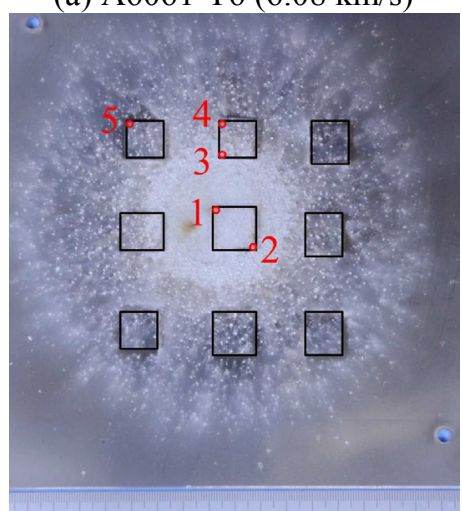

(b) LPSO-Mg $(6.11 \mathrm{~km} / \mathrm{s})$

Fig 3. Photograph of debris cloud impact patterns on aluminum alloy plate as pressure wall after impact.

\section{Conclusions}

The debris cloud of LPSO-Mg was significantly darker than that of A6061-T6. In particular, the ejecta veil of LPSO-Mg was darker than that of A6061-T6. The velocity of the front element for LPSO-Mg was smaller than that for A6061-T6.

Debris cloud impact patterns showed a white circle in the center of the aluminum alloy plates placed behind the target in both cases. In the case of aluminum alloy, we can see clear radial lines, whereas the radial lines become less clear in the case of LPSO-Mg. It was found from the EPMA analysis that debris cloud impact materials of LPSO-Mg target included much magnesium.

This study was supported by ISAS, JAXA as a collaborative program with the Hypervelocity Impact Facility (the Space Plasma Laboratory) and was supported in part by JSPS KAKENHI Grant Number JP24360351, Grant-in-Aid for Scientific Research (B).

\section{References}

1. R. Destefanis, F. Schäfer, M. Lambert, M. Faraud, Int. J. Impact Engineering, 33, 1-12 pp. 219-230 (2006)

2. S. Ryan, T. Hedman, E. L. Christiansen, NASA/TM-2009-214793 (2009)

3. S. Ryan, E. L. Christiansen, NASA/TM-2015218593 (2015)
4. M. Davidson, S. Roberts, G. Castro, R.P. Dillon, A. Kunz, H. Kozachkov, M.D. Demetriou, W.L. Johnson, S. Nutt, D.C. Hofmann, Advanced Engineering Materials, 15(1-2), pp. 27-33 (2013)

5. A. Klavzar, M. Chiroli, A. Jung, B. Reck, Procedia Engineering, 103 pp. 294 - 301( 2015 )

6. N. Kawai, Y. Harada, M. Yokoo, T. Atou, K.G. Nakamura, K. Kondo, Int. J. Impact Engineering, 35(12), pp. 1612-1615 (2008)

7. X. Huang, Z. Ling, Z.D. Liu, H.S. Zhang, L.H. Dai, Int. J. Impact Engineering, 42 pp. 1-10 (2012)

8. L. Hamill, S. Roberts, M. Davidson, W.L. Johnson, S. Nutt, D. C. Hofmann, Advanced Engineering Materials, 16(1) pp. 85-93 (2014)

9. D.C. Hofmann, L. Hamill, E. Christiansen, S. Nutt, Advanced Engineering Materials, 17(9) pp. 13131322 (2015)

10. E. Abe, Y. Kawamura, K. Hayashi, A. Inoue, Acta. Mater., 50 pp.3845-3857 (2002)

11. Y. Kawamura, S. Yoshimoto, Magnesium Technology 2005, (ed. H.I. Kaplan), TMS, pp. 499502 (2005)

12. A.J. Piekutowski, Radiographic Studies of Impact Fragmentation, in High-Pressure Shock Compression of Solids II, Edited by Davison, Lee, Grady, Dennis E., Shahinpoor, Mohsen, pp. 150-175 (1996)

13. A.J. Piekutowski, Int. J. Impact Engineering, 20(610) pp. 639-650 (1997)

14. K. Loft, M.C. Price, M.J. Cole, M.J. Burchell, Int. J. Impact Engineering, 56 pp. 47-60 (2013) 
